\title{
ASPECTOS DINÁMICOS DE LA COMPOSICIÓN Y ESTRUCTURA DE LA COMUNIDAD DE INVERTEBRADOS ACUÁTICOS DE LA RAMBLA DEL MORO DESPUÉS DE UNA RIADA (CUENCA DEL RÍO SEGURA: SE DE ESPAÑA)
}

\author{
M. Ortega, M.L. Suárez, M.R. Vidal-Abarca \& L. Ramírez-Díaz \\ Departamento de Biología Animal y Ecología. Facultad de Biología. Universidad de Murcia \\ Campus de Espinardo. 30100.
}

Palabras clave: rambla, perturbación, riadas, invertebrados acuáticos.

\author{
ABSTRACT \\ DINAMIC ASPECTS OF COMPOSITION AND STRUCTURE OF COMMUNITY \\ OF AQUATIC INVERTEBRATES OF THE RAMBLA DEL MORO AFTER A FLOOD \\ (SEGURA RIVER BASIN, SE OF SPAIN)
}

The variation of composition and structure of invertebrate aquatic community was studied during the first ten days following the 10107186 flood in the Rambla del Moro. This situation is compared with data obtained during the 1982-83 hydrological cycle study developed previously in the same place. The flood reduce taxa richness only by a $31.4 \%$ respect to 1982 autumn situation, therefore the alteration of the community is considered moderate. Invertebrates aquatic community structure reacts to the perturbation modifying the dominance values of the species and maintaining a sufficient numbers of taxa. Community resilience is high because the community structure recovers a month later. Finally, some aspects related with the use of ecological indexes describing the structure of communities subjected to periodical perturbations of the environment are discussed together with the adaptative mechanisms of the species to that perturbation regime.

\section{INTRODUCCIÓN}

En las regiones áridas y semiáridas mediterráneas, como es el caso de la Región de Murcia, los sistemas fluviales están sometidos de forma periódica, pero no regular, a importantes perturbaciones provocadas por las extremas oscilaciones de caudal. Los ríos se secan en la época estival y en otono-invierno sufren aumentos extraordinarios de caudal como respuesta a las lluvias torrenciales y cuya consecuencia inmediata son las riadas o avenidas de agua.

Las alteraciones producidas por las riadas sobre las biocenosis acuáticas están escasamente docu-

Limnetica, 7: 11-24 (1991)

(C) Asociación Española de Limnología, Madrid, Spain mentadas en la bibliografía. Cabe destacar la revisión de Hynes (1970), y el trabajo de Bishop (1973) en un río de Malasia; más recientemente, los de Gray (1980, 1981), Gray \& Fisher (1981), Fisher et al. (1982), Fisher \& Grimm (1988) y Grimm \& Fisher (1989) en el Sycamore Creek, del desierto de Sonora (Arizona), que sufre riadas periódicas; TuRCOTTE \& HARPER (1982) en un río de los Andes y Giberson \& Hall (1988), en un río canadiense. En la región mediterránea, Prevot \& Prevot (1986), analizan los efectos de una avenida de agua en el Río Hoyenn Durance (Francia) sobre los invertebrados acuáticos y la importancia de la deriva como vía de restablecimiento de la comunidad y BADRI et al. (1987) para un río de Marruecos, en el mismo sentido. Otros trabajos en relación con avenidas de agua, provocadas bajo 
control experimental de los volúmenes de caudal, para analizar las perturbaciones que provocan sobre la comunidad de invertebrados acuáticos son los de Scullion \& Sinton (1983), Kraft \& MunDAHI (1984), REICE (1985), HOOPER \& OTTEY (1988) y NeCKLES et al. (1990), entre otros.

En los últimos años, se viene estudiando, de forma sistemática, los efectos de las riadas ocurridas en distintos ríos de la Cuenca del Segura, sobre la composición físico-química del agua (ORTEGA et al., 1988), las alteraciones que provocan en la comunidad de invertebrados acuáticos y las vías que siguen para restablecer la comunidad (ORTEGA, 1988 y OrTEgA et al.).

En este artículo se analizan algunos aspectos de la composición y estructura de la comunidad de invertebrados acuáticos de la Rambla del Moro (Cuenca del Río Segura) durante el ciclo hidrológico 1982-83, y las alteraciones producidas por la riada del 7 de octubre de 1986, así como su evolución en los días posteriores a la misma.

\section{MATERIAL Y MÉTODOS}

\section{Área de estudio}

La Rambla del Moro, localizada en el N.E. de la Región de Murcia (fig. 1), es uno de los afluentes más irregulares del Río Segura en relación al caudal de agua que le aporta. La cuenca vertiente posee una superficie de $386,5 \mathrm{~km}^{2}$ y el $90 \%$ de sus cauces, se comportan hidrológicamente como ramblas, habitualmente secos (sólo transportan agua de evacuación muy rápida, cuando se producen fuertes precipitaciones).

La zona de estudio, se sitúa unos $4 \mathrm{~km}$ antes de la desembocadura de la rambla en el Río Segura, donde el agua circula de forma permanente. El lecho está formado por grava, arena, arcilla y limo, con un tapiz vegetal denso en las zonas deposicionales constituído por Chara vulgaris var. trassicaulis, Zannichellia palustris, Cladophora glomerata, Spyrogira sp. y Enteromorpha sp. Tras la riada acaecida el 7 de octubre de 1986, las transformaciones fisonómicas que se produjeron en la Rambla del Moro afectan principalmente al lecho del cauce y al tapiz vegetal (ORTEGA, 1988). Des- de el año 1988, unos 2 km aguas arriba de la estación de muestreo, se encuentra la Presa del Moro, construida para prevención de avenidas.

Durante el período de estudio la anchura del cauce osciló entre 1,5 y 3,6 m y la profundidad de la lámina de agua entre 5 y $60 \mathrm{~cm}$, según el caudal circulante. La temperatura media del agua fue de $20,8^{\circ} \mathrm{C}$ y la velocidad de la corriente osciló entre 20 y $75 \mathrm{~cm} / \mathrm{s}$. En general, las aguas de la Rambla del Moro se pueden clasificar como alcalinas, tamponadas y muy mineralizadas debido, fundamentalmente, a los cloruros provenientes del tipo de sustrato que drena la red fluvial. Las concentraciones de nitrógeno y fósforo, relativamente elevadas, corresponden a aguas eutrofizadas. Los efectos más importantes de la riada sobre las características físico-químicas del agua fueron un aumento considerable de los sólidos en suspensión, la alcalinidad, los nitratos y los fosfatos, así como, una reducción de los valores de conductividad. Excepto los nitratos, todos los parámetros físico-químicos analizados se estabilizaron, a valores considerados habituales, 27 días después de la riada (OrTEgA et al. 1988).

\section{Metodología}

Pasada la onda de crecida del 7 de octubre, y una vez se pudo acceder a la rambla, se tomaron

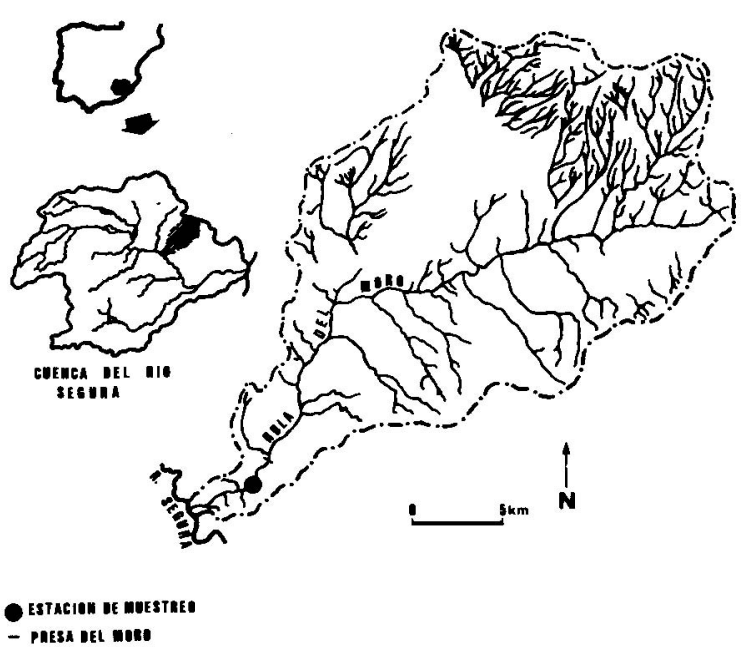

Figura 1. - Cuenca de la Rambla del Moro, área de estudio y lugar de muestreo.

«Rambla del Moro» watershed with location of the study site. 
10 muestras en días consecutivos (del 9 al 18 de octubre de 1986), y otra, 27 días después de la riada (el 3 de noviembre), una vez estabilizado el caudal. La ausencia de una estación de aforo en la Rambla del Moro impidió conocer los módulos absolutos durante la avenida y en los días sucesivos. Se han calculado los módulos relativos mediante la fórmula: Velocidad instantánea de la corriente $\times$ Anchura $\times$ Profundidad de la lámina de agua (MARGALEF, 1983), que quedan reflejados en la figura 2. Las lluvias caídas los días 12 y 16 provocaron dos nuevos picos de caudal los días 13 (396 l/s) y 17-18 de octubre (432 y 420 l/s, respectivamente). Al cabo de 27 días ( $11^{\circ}$ día de muestreo) el caudal de la Rambla del Moro era de $51 \mathrm{l} / \mathrm{s}$.

Para obtener estimas relativas de las poblaciones de invertebrados acuáticos de la Rambla del Moro, se empleó el método de captura por unidad de esfuerzo, con ayuda de una manga triangular de $35 \mathrm{~cm}$ de lado y luz de malla de $1 \mathrm{~mm}$ (SOLER, 1984). Siempre que fue posible, las determinaciones de los invertebrados se han realizado a nivel específico, a excepción del grupo de los dípteros de evidente dificultad taxonómica.

Con el fin de describir algunos aspectos de la organización de la comunidad de invertebrados acuáticos de la Rambla del Moro, se han empleado distintos índices, clásicos en ecología (riqueza, dominancia, diversidad específica, etc.), que permiten salvar la dificultad de realizar un estudio descriptivo de comunidades formadas por muchas poblaciones (MONTES, 1980). Sin embargo, no hay que olvidar que estos índices generales (MAY, 1975), no permiten detectar las complejas relaciones intra e interespecíficas de las poblaciones que integran la comunidad (RICKLEFS, 1979).

Para poder contrastar los resultados obtenidos, se han analizado los datos de invertebrados acuáticos, recogidos en la misma estación de muestreo y con el mismo método en tres épocas distintas del ciclo hidrológico 1982-83 (otoño, invierno y verano), durante el cual las características fisonómicas de la Rambla del Moro fueron muy variables. En otoño, el cauce mantenía zonas erosionales y deposicionales con sustratos de diferente textura y una acumulación de detritos que permitía el asentamiento de grandes manchas de macrófitos sumergidos. En invierno, y tras haber pasado una riada el 20 de octubre, que eliminó completamen- te tanto a los macrófitos como a la comunidad de invertebrados (SUAREZ \& Vidal-ABARCA, com. per.), el cauce mantenía un sustrato fundamentalmente de grava. En verano, el cauce quedó estructurado a modo de charcas aisladas sin comunicación entre sí. Durante el período 19821986, la rambla no sufrió avenidas de carácter catastrófico. La construcción de un embalse de laminación de avenidas (fig. 1), fue posterior a este estudio.

\section{RESULTADOS}

\section{Composición y estructura de la comunidad}

En la tabla 1, se presenta la matriz de abundancia, de las especies de invertebrados acuáticos recogidos en cada uno de los tres períodos considerados (otoño, invierno y verano), durante el ciclo hidrológico 1982-83. En total se contabilizaron 7025 individuos pertenecientes a 57 taxones. Las variaciones de la composición de la comunidad y de la abundancia son notables, debido a la perturbación hídrica (riada de octubre de 1982), que afectó a la comunidad. Los heterópteros y coleópteros, son muy abundantes seguidos de los dípteros y efímeras. El resto de grupos (odonatos, moluscos, crustáceos e hidrácaros), son menos importantes durante el ciclo considerado.

La riqueza faunística de la comunidad en la muestra de otoño (42 taxones), es la mayor de las tres analizadas (tabla 2), así como la de mayor abundancia de individuos (4163). Los heterópteros y coleópteros aportan mayor riqueza específica (11 y 10 taxones, respectivamente).Las especies de coleópteros con mayor densidad de población son Berosus sp. y Potamonectes cerisyi, ambas muy eurioicas, y características de aguas remansadas con sustrato arenoso y abundante vegetación. Los odonatos, con 7 especies, representan un 15,91\% de la riqueza total, la mayor registrada para este grupo, coincidiendo con la máxima riqueza taxonómica de la comunidad en todo el estudio. A pesar de ello, su contribución a la abundancia total de individuos, es tan sólo del 1,15\%.

En invierno, debido tanto al empeoramiento habitual de las condiciones ambientales, como a 


\begin{tabular}{|c|c|c|c|c|}
\hline Codrgo & Taxones & $\begin{array}{l}\text { Otoño } \\
3-9-82\end{array}$ & $\begin{array}{l}\text { Invierno } \\
27-2-83\end{array}$ & $\begin{array}{l}\text { Verano } \\
3-7-83\end{array}$ \\
\hline$m 1$ & Mercuria confusa & 77 & & 87 \\
\hline hd & HYDRACARINA & 2 & . & 1 \\
\hline cr & Echinogammarus sp. & 5 & 3 & 146 \\
\hline el & Cloeon dipterum & 4 & 53 & 423 \\
\hline $\mathrm{e} 2$ & Caenis luctuosa & 1 & 2 & 11 \\
\hline ol & Coenagrion caerulescens & 54 & . & 3 \\
\hline 02 & Coenagrion mercuriale & 37 & . & - \\
\hline 03 & Ischnura elegans & 13 & . & 5 \\
\hline o4 & Anax imperator & 17 & . & 9 \\
\hline 05 & Crocothemrs erythraea & 3 & . & 17 \\
\hline 06 & Orthetrum brunneum & 10 & . & . \\
\hline 07 & Orthetrurn coerulescens & 5 & 2 & 1 \\
\hline h6 & Mesovelia vittigera & 12 & & . \\
\hline h15 & Hydrometra stagnorum & & & \\
\hline \multirow[t]{2}{*}{ h14 } & Gerris thoracicus & 11 & & \\
\hline & Micronecra scholtzi & 97 & & \\
\hline h17 & Cymntia rogenhoferi & 76 & & \\
\hline h16 & Heliocorisa vermiculata & 307 & & \\
\hline h9 & Sigara scrrpta & 37 & & \\
\hline h2 & Naucoris maculatus & 1.303 & & 19 \\
\hline h3 & Nepa cinerea & 17 & & 1 \\
\hline h1 & Anisops debilis perplexa & 264 & & 1 \\
\hline h18 & Notonecta maculata & 2 & & 3 \\
\hline h4 & Plea minutissima & 336 & & 1 \\
\hline $\mathrm{c} 17$ & Orectochilus villosus & 1 & & \\
\hline $\mathrm{c} 12$ & Haliplus lineaticollis & & 11 & 7 \\
\hline c16 & Laccophilus hyalinus & & & 3 \\
\hline c15 & Bidessus minutissimus & 3 & & \\
\hline c6 & Hydroglyphus signatellus & 57 & & \\
\hline $\mathrm{c} 11$ & Yola bicarinata & 2 & & \\
\hline $\mathrm{c} 4$ & Herophydrus musicus & 5 & & \\
\hline $\mathrm{c1}$ & Potamonectes cerisyi & 139 & 18 & 260 \\
\hline c18 & Agabus sp. & & 2 & 3 \\
\hline $\mathrm{c} 19$ & Rhantus pulverosus & & 1 & 1 \\
\hline c3 & Ochthebius sp. & 30 & 3 & 200 \\
\hline$c 2$ & Berosus sp. & 949 & 10 & 50 \\
\hline$c 5$ & Laccobius sp. & 6 & 8 & 3 \\
\hline$c 7$ & Enochrus sp. & 85 & 8 & 320 \\
\hline c10 & Coelostoma sp. & & & 4 \\
\hline$c 13$ & Dryops sp. & & & 3 \\
\hline d5 & Culex pipiens & & 7 & 1 \\
\hline $\mathrm{d} 7$ & Culex sp. & & 4 & 2 \\
\hline d4 & Simuliinae & 6 & 290 & \\
\hline d5 & Chelocnethasp. & 1 & 28 & \\
\hline d6 & Tanypodinae & 6 & 63 & 2 \\
\hline d7 & Orthocladiinae & 1 & 548 & 17 \\
\hline $\mathrm{d} 8$ & Chrronomini & & 2 & . \\
\hline d 9 & Chironomus gr. plumosus & & . & 4 \\
\hline $\mathrm{d} 10$ & Tanytarsini & 16 & 7 & 2 \\
\hline d11 & $\begin{array}{l}\text { Ceratopogonidae gr. Dasy- } \\
\text { helea }\end{array}$ & 153 & 29 & 1 \\
\hline $\mathrm{d} 12$ & Id. gr. Vermiforme & & & . \\
\hline $\mathrm{d} 13$ & Stratiomys sp. & & & 33 \\
\hline $\mathrm{d} 14$ & Odontomyia sp. & & & 9 \\
\hline $\mathrm{d} 15$ & Atalantinae & & 12 & \\
\hline $\mathrm{d} 16$ & Tabanus sp. & 4 & & \\
\hline $\mathrm{d} 17$ & Ephydra sp. & 7 & & 88 \\
\hline d18 & Melachonelia sp. & & & \\
\hline
\end{tabular}

la gran avenida sufrida en octubre de 1982, se redujo la composición faunística a 25 taxones y el número total de individuos a 1120. Es de resaltar la ausencia de dos grupos taxonómicos, los hidrácaros, y los heterópteros que pasan de ser el grupo más abundante y con mayor riqueza específica, a no aparecer ningún ejemplar. Los heterópteros, en general, son un grupo pionero, con gran capacidad de vuelo que les permite, en situaciones de inestabilidad, buscar y explotar nuevos ambientes (Millan, 1985). La comunidad de invierno está caracterizada por la gran abundancia de dípteros $(89,10 \%)$, con una riqueza específica de 12 taxones. Las familias más abundantes (tabla 1), son los quironómidos, y, en especial, los ortocladinos, típicamente reófilos, y los simúlidos, característicos de las aguas corrientes.

En la muestra de verano se observa un restablecimiento parcial de la comunidad que aumenta su composición faunística (37 taxones), con respecto a la de invierno. Los coleópteros son los que presentan una mayor riqueza de especies (11 taxones) y abundancia (854 individuos). Los taxones mayoritarios son Enochrus sp., Potamonectes cerisyi y Ochthebius sp., característicos de pequeños cuerpos de agua con alto grado de salinidad y abundante vegetación. Aparecen otros nuevos como Laccophilus hialinus y Coelostoma sp. con marcado carácter halófilo y Dryops sp. habitante típico de la ecofase anfibia de pozas y charcas. Los dípteros pierden importancia disminuyendo su riqueza taxonómica (10 taxones) y abundancia $(9,13 \%)$, al hacerlo la población de quironómidos. Los efídridos, típicos habitantes de aguas salinas, son los más abundantes en este caso. Los heterópteros vuelven a estar presentes con 26 individuos pertenecientes a 6 especies, todos nepomorfos a excepción de Hydrometra stagnorum (semiacuático).

En definitiva, las variaciones de la composición y riqueza faunística en la Rambla del Moro, a lo largo del ciclo hidrológico 1982-83, son debidas tanto al cambio de caudal y velocidad del agua (es-

Tabla 1. - Matriz de abundancia total ( $\mathrm{n}^{\circ}$ individuos/unidad de esfuerzo) para cada muestra (otoño, invierno y verano), del ciclo hidrológico 1982-83.

Total abundance values matrix of the autumn, winter and summer samples in the 1982-83 hydrological cycle. 
tacionalidad), como a las alteraciones puntuales (avenidas), que producen la modificación de las características fisonómicas del tramo estudiado.

Teniendo en cuenta que el número total de especies es limitado en una comunidad y que sus poblaciones no son igualmente abundantes, éstas se pueden seriar, por orden de abundancia, de la más numerosa a la más rara (MARGALEF, 1977). La abundancia relativa o dominancia de las especies, se utiliza como un índice para describir aspectos estructurales de la comunidad (Montes, 1980). En la figura 3 , se presentan las curvas obtenidas para las tres muestras consideradas en el ciclo hidrológico 1982-83. La correspondiente al otoño, sigue una distribución log-serie (RoutledGe, 1980), indicando la presencia de una comunidad considerada «estable», faunísticamente rica y con un elevado número de individuos, aunque dominada por un par de especies Naucoris maculatus y Berosus $s p$.

La curva de invierno, posee un pico muy pronunciado, llegando a un valor de abundancia relativa de 48,9\% para los ortocladinos, seguidos de los simúlidos. No obstante, al existir problemas para la identificación de las larvas de los dípteros, el ajuste de la curva podría variar adquiriendo mayor importancia los quironómidos. Los grandes intervalos de abundancia relativa observados, junto al ajuste de la curva a una serie geométrica (WHITTAKER, 1975), dan idea de la pobreza y fragilidad de la comunidad de invierno.

En la muestra de verano, la comunidad está dominada por Cloeon gr. dipterum $(24,28 \%)$, seguida de Enochrus sp., Potamonectes cerisyi, y Octhebius sp., en un rango de abundancia entre $19 \mathrm{y}$ $11 \%$. En general, la comunidad posee una gran riqueza, con numerosas especies de rangos intermedios. El ajuste de la curva a una distribución log-serie sugiere un cierto grado de estabilidad de la comunidad.

En la tabla 2, se presentan los valores de diversidad específica (Shannon \& Weaver, 1949), máxima o teórica y la equidad, obtenidas para cada una de las muestras recogidas en el ciclo hidrológico 1982-83. La muestra de otoño proporciona el mayor valor de diversidad específica. Sin embargo, la equidad con un valor de $61 \%$, delata la presencia de unas pocas especies de heterópteros dominantes que han encontrado las condiciones óptimas para el desarrollo de sus

Tabla 2. - Parámetros físico-químicos, riqueza específica, abundancia y diversidad para cada muestra (otoño, invierno y verano), del ciclo hidrológico 1982-83.

Physical and chemical parametres, specific richness, abundance and diversity for autumn, winter and summer samples during 1982-83.

\begin{tabular}{|c|c|c|c|c|c|c|}
\hline & \multicolumn{2}{|c|}{ Otoño } & \multicolumn{2}{|c|}{ Invierno } & \multicolumn{2}{|c|}{ Verano } \\
\hline & $n .^{\circ}$ taxones & ahund (\%) & n." taxones & abund (\%) & n." taxones & ahund (\%) \\
\hline Moluscos & 1 & 1,85 & 1 & 0,10 & 1 & 5 \\
\hline Hidrácaros & 1 & 0,05 & - & - & 1 & 0.06 \\
\hline Crustáceos & 1 & 0,12 & 1 & 0.27 & 1 & 8,38 \\
\hline Efímeras & 2 & 2,20 & 2 & 4.90 & 2 & 24,91 \\
\hline Odonatos & 7 & 1,15 & 1 & 0,18 & 5 & 2,01 \\
\hline Heterópteros & 11 & 59,14 & - & - & 6 & 1.49 \\
\hline Coleóptcros & 10 & 30,77 & 8 & 5,45 & 11 & 49.02 \\
\hline Dípteros & 9 & 4.72 & 12 & 89.10 & 10 & 9,13 \\
\hline Total taxones & 42 & & 25 & & 37 & \\
\hline Diversidad (H) & \multicolumn{2}{|c|}{3,4} & \multicolumn{2}{|c|}{2,8} & \multicolumn{2}{|c|}{3,3} \\
\hline Diversidad Máxima (Hmax) & \multicolumn{2}{|c|}{5,5} & \multicolumn{2}{|c|}{4,9} & \multicolumn{2}{|c|}{5.3} \\
\hline Equidad \% (E) & \multicolumn{2}{|c|}{61} & \multicolumn{2}{|c|}{57} & \multicolumn{2}{|c|}{63} \\
\hline \multicolumn{7}{|l|}{ Variables físico-químicas } \\
\hline Conductividad $\mu \mathrm{S} / \mathrm{cm}$ & \multicolumn{2}{|c|}{13500} & \multicolumn{2}{|c|}{9200} & \multicolumn{2}{|c|}{12000} \\
\hline S. Suspensión mg/l & \multicolumn{2}{|c|}{1,27} & \multicolumn{2}{|c|}{28,65} & \multicolumn{2}{|c|}{49,28} \\
\hline Veloc. corriente $\mathrm{cm} / \mathrm{s}$ & \multicolumn{2}{|c|}{15} & \multicolumn{2}{|c|}{25} & \multicolumn{2}{|c|}{0} \\
\hline Profundidad $\mathrm{cm}$ & \multicolumn{2}{|c|}{25} & \multicolumn{2}{|c|}{60} & \multicolumn{2}{|c|}{20} \\
\hline
\end{tabular}


Tabla 3. - Matriz de abandancia total ( $\mathrm{n}^{\circ}$ de individuos/unidad de esfuerzo), para cada una de las 11 muestras tomadas después de la riada del 7 de octubre de 1986.

Total abundance values matrix of the 11 day samples after the flood at october 7 th, 1986 .

\begin{tabular}{|c|c|c|c|c|c|c|c|c|c|c|c|c|}
\hline \multirow[b]{2}{*}{ Codigo } & \multirow[b]{2}{*}{ Taxones } & \multicolumn{11}{|c|}{ Muestras } \\
\hline & & $\begin{array}{c}I \\
9-10-86\end{array}$ & $\begin{array}{c}2 \\
10-10-86 \\
\end{array}$ & $\begin{array}{c}3 \\
11-10-86\end{array}$ & $\begin{array}{c}4 \\
12-10-86\end{array}$ & $\begin{array}{c}5 \\
13-10-86\end{array}$ & $\begin{array}{c}6 \\
14-10-86\end{array}$ & $\begin{array}{c}7 \\
15-10-86\end{array}$ & $\begin{array}{c}8 \\
16-10-86\end{array}$ & $\begin{array}{c}9 \\
17-10-86\end{array}$ & $\begin{array}{c}10 \\
18-10-86\end{array}$ & $\begin{array}{c}11 \\
3-11-86 \\
\end{array}$ \\
\hline m 1 & Mercuria confusa & 3 & 6 & 9 & 5 & 5 & 6 & 13 & 7 & 5 & 23 & 2 \\
\hline cr & Echinogammarus sp. & 8 & 3 & 6 & 2 & . & 6 & 6 & 15 & 15 & 15 & 5 \\
\hline e3 & Centroptilum pennulatum & . & & & . & . & . & . & . & 1 & . & . \\
\hline e1 & Cloen dipterum & 5 & 24 & 13 & 9 & 4 & 9 & 6 & 4 & 3 & 7 & 5 \\
\hline e6 & Colen simile & . & . & . & . & . & . & . & 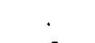 & 1 & . & . \\
\hline e2 & Caenis luctuosa & . & . & . & 1 & & 1 & . & 2 & & 2 & . \\
\hline 01 & Coenagrion caerulescens & 185 & 45 & 58 & 107 & 12 & 131 & 49 & 46 & 47 & 129 & 10 \\
\hline 02 & Coenagrion mercuriale & 37 & 19 & 23 & 23 & 11 & 3 & 20 & 13 & 2 & . & . \\
\hline 03 & Ischnura elegans & 17 & 6 & 18 & . & . & 14 & 4 & . & 6 & 6 & . \\
\hline 04 & Anax imperator & 1 & 1 & 6 & 1 & . & . & 1 & & . & 2 & 2 \\
\hline 05 & Crocothemis erythraea & $\cdot$ & 1 & . & $\cdot$ & . & . & 1 & 1 & . & 1 & . \\
\hline 06 & Orthetrum brunneum & 1 & 2 & 4 & 1 & . & . & 1 & 4 & 2 & 10 & 2 \\
\hline 07 & Orthetrum coerulescens & 3 & 2 & 2 & 4 & 1 & 1 & 6 & 4 & . & 2 & 3 \\
\hline h6 & Mesovelia vittigera & . & . & & . & 1 & . & 1 & . & . & . & . \\
\hline h15 & Hydrometra stagnorum & . & . & . & . & . & . & 1 & . & . & . & . \\
\hline h5 & Hebrus pusillus & . & . & . & . & 1 & . & . & . & . & 1 & . \\
\hline h7 & Microvelia pigmaea & & . & 2 & . & . & . & . & 1 & . & . & . \\
\hline h14 & Gerris thoracicus & . & 1 & . & . & . & . & . & 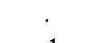 & . & . & . \\
\hline h11 & Micronecta scholtzi & . & & . & . & & 1 & . & 1 & . & . & . \\
\hline h10 & Sigara scripta & . & . & 1 & 3 & 2 & 1 & . & . & . & . & . \\
\hline h2 & Naucoris maculatus & 19 & 14 & 10 . & 18 & 3 & 15 & 13 & 13 & 4 & 9 & 10 \\
\hline h3 & Nepa cinerea & . & . & . & 1 & & 1 & . & 3 & 1 & 4 & 1 \\
\hline h4 & Plea minutissima & & 5 & 6 & 3 & . & 2 & 4 & . & . & 3 & . \\
\hline c12 & Haliplus lineaticollis & . & 1 & . & . & . & . & 1 & . & . & . & . \\
\hline c9 & Noterus laevis & . & . & 2 & . & 1 & . & 1 & . & 1 & . & \\
\hline c16 & Laccophilus hyalinus & . & . & . & 1 & . & . & . & . & . & . & . \\
\hline c15 & Bidessus minutissimus & . & . & 1 & 1 & . & . & . & . & . & . & . \\
\hline c6 & Hydroglyphus signatellus & 1 & . & 2 & 1 & . & 1 & 3 & 3 & 1 & 1 & \\
\hline $\mathrm{c} 11$ & Yola bicarinata & . & . & . & . & . & . & 1 & . & . & 1 & . \\
\hline $\mathrm{c4}$ & Herophydrus musicus & . & 3 & . & . & . & 1 & . & 1 & & . & . \\
\hline $\mathrm{cl}$ & Potamonectes cerisyi & 7 & 29 & 16 & 27 & 17 & 12 & 36 & 12 & 10 & 13 & 2 \\
\hline c3 & Ochthebius sp. & 4 & 12 & 5 & 4 & 3 & 4 & 5 & 7 & 7 & 5 & 1 \\
\hline$c 2$ & Berosus sp. & 10 & 42 & 13 & 42 & 6 & 5 & 2 & 5 & 5 & 12 & 12 \\
\hline c5 & Laccobius sp. & 3 & 3 & 2 & 1 & 1 & 2 & 2 & . & 1 & 1 & 2 \\
\hline c14 & Helochares sp. & . & 1 & . & . & . & . & . & . & . & & . \\
\hline c7 & Enochrus sp. & 6 & 4 & 2 & 2 & 2 & . & 1 & 2 & 3 & 4 & . \\
\hline c8 & Hydrous piceus & 1 & . & & . & . & . & . & . & & & \\
\hline c13 & Dryops sp. & . & 1 & 1 & 1 & . & 1 & . & 1 & & . & . \\
\hline $\mathrm{d} 1$ & Tipulidae (ninfa) & . & . & . & . & . & . & . & . & . & 1 & \\
\hline d4 & Simuliinae & 5 & 2 & 3 & . & . & 2 & . & & 1 & 2 & \\
\hline d5 & Chelocnetha sp. & . & & . & . & . & . & . & 1 & & . & \\
\hline d6 & Tanypodinae & 2 & 1 & . & . & 1 & . & . & & 1 & 1 & 1 \\
\hline d7 & Orthocladiinae & . & & & . & 1 & 3 & 1 & 1 & 8 & 7 & 6 \\
\hline d8 & Chironomini & & & . & . & . & . & . & . & 2 & 2 & . \\
\hline d10 & Tanytarsini & 5 & 2 & . & . & 2 & 3 & 4 & 13 & 17 & 15 & 11 \\
\hline d11 & Ceratopogonidae gr. Dasyhelea & . & 1 & . & 1 & 3 & $\cdot$ & 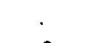 & & . & 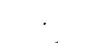 & 5 \\
\hline d12 & Ceratopogonidae gr. Vermiforme & . & . & . & . & . & . & 2 & . & 6 & 1 & 3 \\
\hline d13 & Stratiomys sp. & 1 & 1 & . & 1 & . & 6 & 5 & 1 & 1 & 3 & . \\
\hline d14 & Odontomyia sp. & & . & . & 1 & . & . & . & & . & 1 & . \\
\hline d16 & Tabanus sp. & 2 & . & . & . & . & . & . & . & . & 2 & . \\
\hline d17 & Ephydra sp. & . & 2 & . & 1 & 1 & 1 & 5 & 1 & . & 1 & 2 \\
\hline
\end{tabular}


poblaciones. Por el contrario, la comunidad de invierno, muestra los valores más bajos de diversidad específica y máxima $(2,8$ y 4,9 , respectivamente), consecuencia de los efectos de la nada de octubre de 1982, que uniformizó las características ambientales del cauce. En verano, los valores de diversidad específica y máxima aumentan, siendo muy parecidos a los de otoño, aunque la equidad sea mayor $(63 \%)$. Esto, muestra una comunidad con las relaciones de dominancia menos importantes, influenciado, quizás, por el hecho de que el cauce queda estructurado en pozas aisladas, sin flujo entre ellas, donde se concentran las poblaciones y las relaciones interespecíficas son más estrechas.

\section{Variación temporal después de la riada de 1986}

En la tabla 3, se presenta la matriz de abundancia de las especies correspondientes a las muestras tomadas durante 10 días consecutivos después de la riada de 1986, y a la muestra 11 , tomada 27 días después de la onda de crecida. Para describir y valorar el efecto producido por esta riada sobre la comunidad de invertebrados acuáticos, los resultados se comparan con los obtenidos en la misma época del año 1982, antes de producirse la avenida de agua.
Durante los diez días después de la riada se recolectaron un total de 2132 individuos, pertenecientes a 51 taxones diferentes. De ellos, 37 son comunes con los recogidos en la muestra de otoño de 1982, por tanto la reducción en el número de taxones es del 31,4\% (sin considerar los 16 taxones que aparecen esporádicamente en alguna de las muestras). Jones, (1951) y McLAy, (1968), obtienen una reducción en la mitad del número de taxones en las comunidades de dos ríos de Inglaterra y Nueva Zelanda, tras una crecida; GAsCHIGNARD (1984), anota una reducción del $75 \%$ del número de taxones en un afluente del Ródano en iguales circunstancias y GraY (1980), estima una reducción del $80-100 \%$ de la fauna del bentos en un río del desierto de Arizona, por lo que, el impacto de la riada de octubre de 1986, en el área de estudio, sobre la riqueza taxonómica, puede considerarse mínimo.

Hydracarina, Cymatia rogenhoferi, Heliocorixa vermiculata, Anisops debilis perplexa, Notonecta maculata y Orectochilus villosus, encontrados en la comunidad de otoño, y ausentes todos los días de muestreo tras la riada, serían los taxones más afectados por el aumento de caudal. Prácticamente, todos ellos son predadores, y la posibilidad de retorno al tramo del cauce del que han sido desplazados por la crecida, dependerá tanto de su ca-

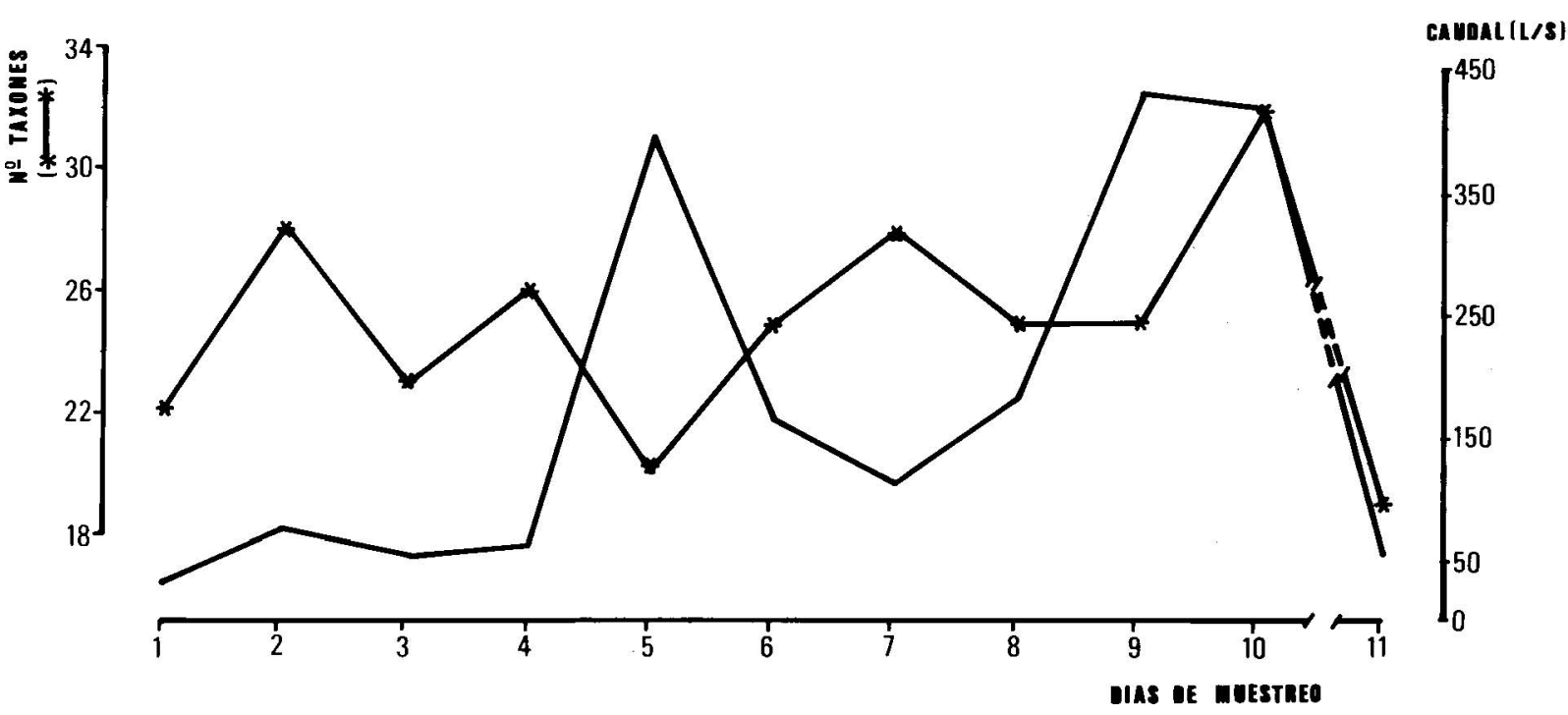

Figura 2. - Evolución de la riqueza taxonómica y del caudal durante el período de muestreo, después de la riada del 7 de octubre de 1986.

Daily changes in the taxa richness and discharge during the sampling period, after the flood at october the 7th, 1986. 
pacidad de dispersión como del grado de organización y equilibrio de la comunidad (MINSHALL \& Petersen, 1985).

Los taxones que aparecen esporádicamente en las 10 muestras después de la riada de 1986 y que no forman parte de la comunidad encontrada durante el ciclo 1982-83, ni de la muestra 11 (Centroptilum pennulatum, Cloeon simile, Hebrus pusillus, Microvelia pygmaea, Noterus laevis, Helochares $s p$, Hydrous pisceus, y tipúlidos), podrían proceder, por deriva, de zonas de cauce situadas aguas arriba del tramo de estudio o de otros cuerpos de agua cercanos a él.

Durante los diez días considerados, la riqueza taxonómica sufre moderadas oscilaciones, desde 20 el $5^{\circ}$ día hasta 32 el 10" que, a grandes rasgos, responde en sentido opuesto a las oscilaciones de caudal (figura 2). De hecho, con el primer pico de caudal ( $5^{\circ}$ día de muestreo), se registra el primer descenso brusco del número de taxones (20), siendo el mínimo del estudio. La disminución sufrida como consecuencia del segundo máximo de caudal, no llegó a ser tan brusca, contabilizándose hasta 25 taxones el 9. día de muestreo. En la muestra $11 .^{a}$, la composición faunística de la comunidad, formada por 19 taxones, parece mostrar una tendencia a mantener poblaciones capaces de tolerar las fluctuaciones del caudal (fig. 2).

Considerando la riqueza de especies, agrupada por órdenes, para cada uno de los días de muestreo, los coleópteros son los que poseen una mayor riqueza a excepción del 2" y 10" días, que lo fueron los odonatos y dípteros, respectivamente. En ambas ocasiones, los coleópteros fueron el segundo grupo en importancia. Este hecho también es constatado por FISHER et al. (1982), quienes encuentran que el menor porcentaje de pérdidas después de una riada se produce en la taxocenosis de coleópteros y heterópteros.

Respecto a la frecuencia de aparición de los distintos taxones en las diez muestras, 18 se pueden considerar «frecuentes» $(100-66,6 \%)$, y todos aparecen en la comunidad de otoño de 1982, aunque sólo 12 de ellos permanecen el 11" día de muestreo. En esta categoría, existen representantes de todos los órdenes taxonómicos. De los 12 taxones con una «frecuencia media» $(66,6-$ $33,3 \%$ ), dos (Noterus laevis y Dryops sp.) no aparecen en otoño del 82, y cinco aparecen en la muestra $11^{\mathrm{a}}$. Los 21 taxones considerados como «poco frecuentes* $(33,3-0 \%)$, resultan ser los más abundantes y, de ellos, sólo uno se recogió el $11^{\circ}$ día, y 13 no aparecen en otoño del 82. En este grupo destacan los coleópteros (7 taxones), y los dípteros y heterópteros (6 cada uno).

Si se considera la frecuencia de aparición de órdenes taxonómicos, los odonatos son los que presentan una mayor proporción de poblaciones frecuentes ( 5 de las 7 especies encontradas poseen una frecuencia superior al $70 \%$ ). Los dípteros y heterópteros presentan 6 taxones de un total de 13 y 10 , respectivamente, con una frecuencia de aparición igual o menor al $30 \%$, por lo que se pueden considerar como especies «fugaces». Los coleópteros destacan por incluir especies cuya frecuencia se sitúa en los extremos del rango (6 taxones son frecuentes y 7 poco frecuentes).

El análisis de las curvas de dominancia o abundancia relativa de cada especie en las 11 muestras analizadas (fig. 4), permite establecer una secuencia, a modo de ciclo, que se repite en dos ocasio-

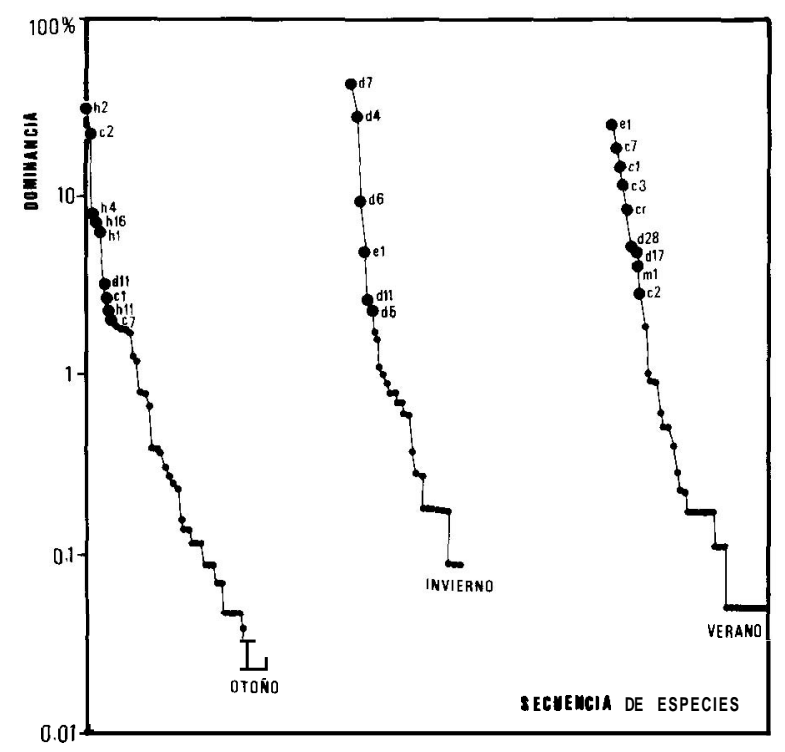

Figura 3. - Curvas de abundancia relativa o dominancia de los taxones recogidos en cada una de las muestras del ciclo hidrológico 1982-83 (los códigos de los taxones se encuentran en la tabla 1).

Relative abundance curves of the taxa collected in autumn, winter and summer samples during 1982-83 (key to species codes can be found in table 1). 
nes en los 10 primeros días. Partiendo de los días 1,6 y 10 del muestreo, posteriores a los picos de caudal (onda de crecida del 7 de octubre, el 5." y el 9." día de muestreo), se presenta una especie muy dominante que es la misma en las tres comunidades, (Coenagrion caerulescens), con una abundancia relativa del $56.7 \%, 56,4 \%$ y $44,9 \%$, respectivamente. Esta especie, provoca un pico inicial de las curvas que descienden bruscamente hasta alcanzar una especie más, que se puede considerar dominante, en cada una de ellas: Coena- grion mercuriale, Naucoris maculatus y Mercuria confusa, respectivamente. A continuación, las curvas caen hasta alcanzar unos porcentajes de abundancia relativa que oscilan entre 3 y $5 \%$, a partir de los cuales el descenso es menos brusco y engloba a la mayoría de las especies, que son las poco dominantes.

En esta secuencia, una segunda fase sería la representada por las curvas de dominancia correspondientes a los días 2 y 7 . En ellas desaparece el pico acusado, descendiendo más moderamente,

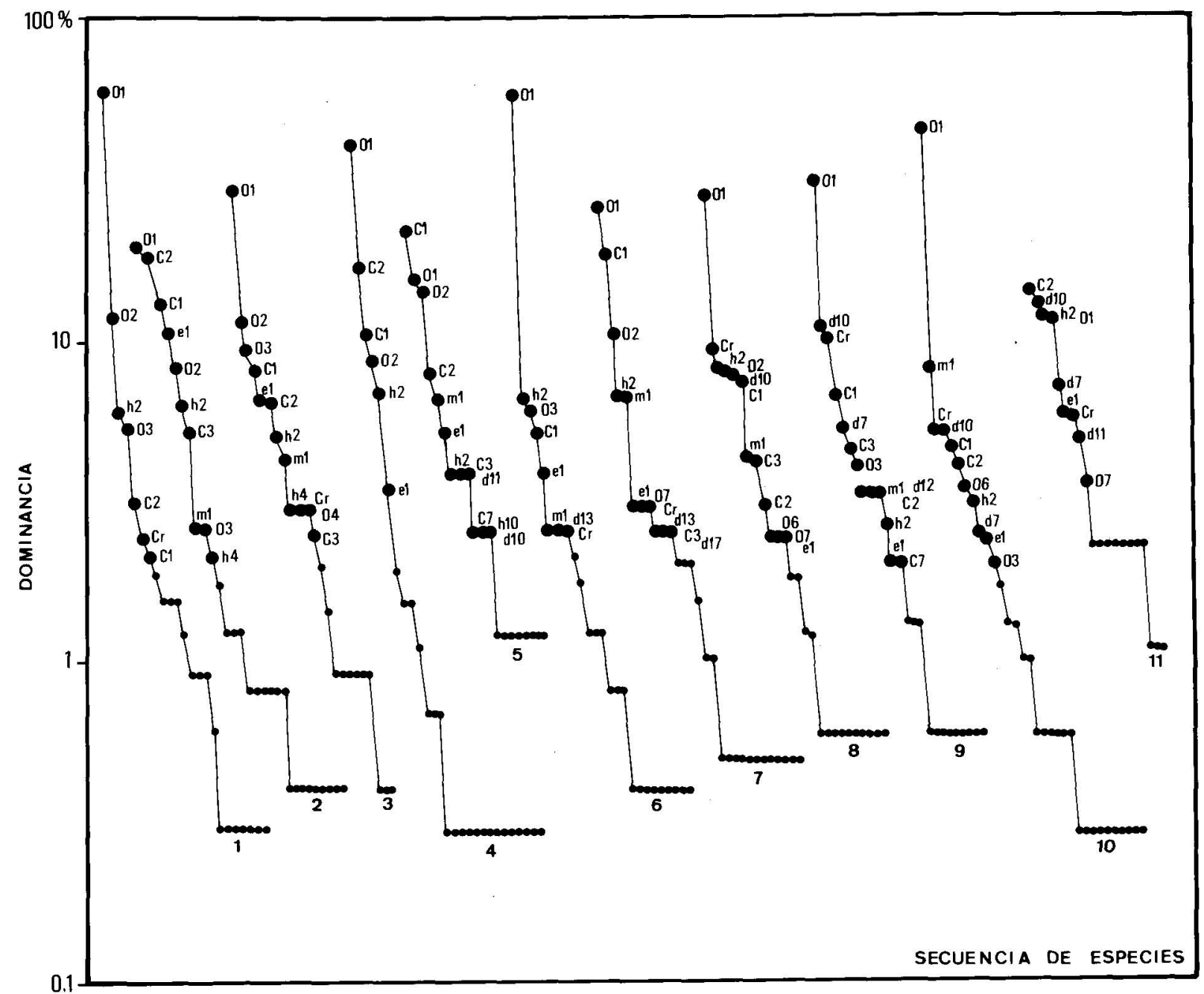

Figura 4. - Curvas de abundancia relativa o dominancia para cada una de las 11 muestras tomadas después de la riada de 1986 (los códigos de los taxones se encuentran en la tabla 3 ).

Relative abundance curves of the taxa collected in the 11 days following the flood of 1986 (key to species codes can be found in table 3). 
por el acercamiento de las especies dominantes, manteniéndose las «colas» con presencia de numerosos taxones.

A continuación, las curvas de dominancia de los días 3 y 8 , muestran comunidades en las que una especie dominante provoca un pico inicial, al igual que en los días 1, 6 y 10 aunque de menor magnitud. Junto a esta especie se encuentran cinco

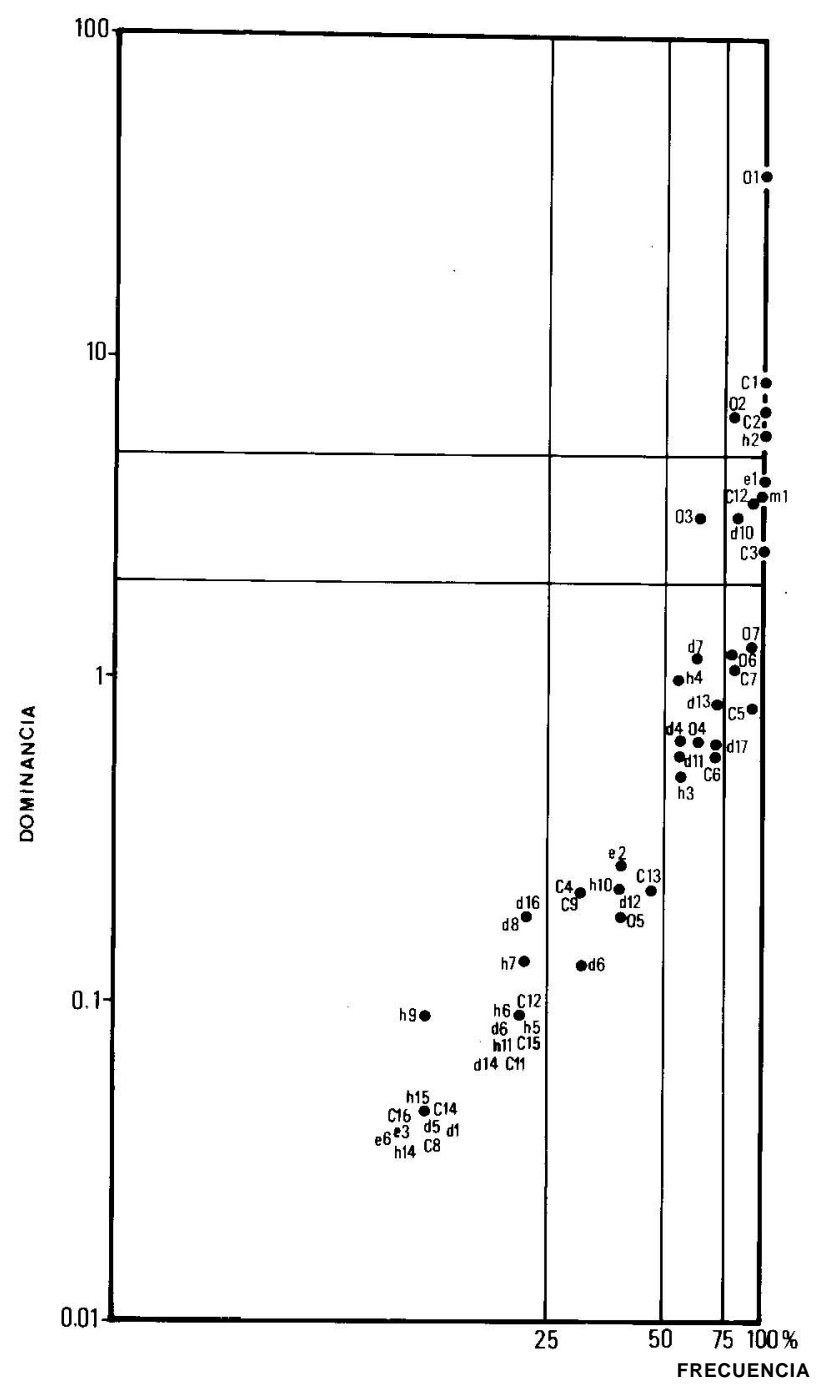

Figura 5. - Valores de frecuencia y dominancia de los taxones encontrados en los 11 días después de la riada de 1986 (los códigos de los taxones se encuentran en la tabla 3 ).

Frequency and dominance values of taxa collectcd in the 11 days following the flood of 1986 (key to species codcs can be found in table $\mathbf{3}$ ). taxones dominantes en cada comunidad, amortiguando la caída de las curvas que, en este caso, reflejan una reducción del número de especies poco dominantes en la «cola».

El resto de las curvas, evoluciona reflejando la existencia de cambios continuos en la estructura de la comunidad (días 4 y 5) o distribuyéndose los rangos de dominancia de las especies más uniformemente (día 9).

La curva correspondiente al 11" día, muestra una mayor constancia de proporciones entre los valores de dominancia de las especies, probablemente, como consecuencia del cese de las oscilaciones de caudal y no como indicativo del mayor grado de organización de la comunidad.

La evolución de las curvas refleja cambios continuos en la estructura de la comunidad como consecuencia de la perturbación creada por los picos de caudal posteriores a la onda de crecida. La llegada y salida continua de especies observada «in situ», desestabiliza continuamente la comunidad (ORTEGA et al., 1991).

Para obtener una idea aproximada sobre la capacidad de adaptación que poseen las especies a las condiciones fluctuantes del sistema (SANTOS, 1983), se puede recurrir a representar conjuntamente los valores de dominancia y frecuencia de aparición de cada una de ellas en el conjunto de las muestras.

En la figura 5, se presenta la posición de los taxones en función de los valores de estos dos índices, en escalas logarítmicas, obteniéndose una clasificación de las especies en cinco categorías (THIERY, 1978): especies fundamentales (frecuencia $>50 \%$ y dominancia $>10 \%$ ); constantes (frecuencia $>50 \%$ y dominancia $<10 \%$ ); acompañantes (frecuencia 25-50\%); accesorias (frecuencia 13-25\%) y esporádicas (frecuencia $<13 \%$ ).

Unicamente, Coenagrion caerulescens, se presenta como especie fundamental. FisHer et al. (1982), encuentran que los odonatos junto con las efímeras, ante una perturbación ambiental importante, siguen un comportamiento característico que consiste en acelerar el proceso reproductor, efectuando gran número de puestas de las que emergen larvas de rápido crecimiento que disminuyen, en gran medida, el tiempo de duración de su ciclo de vida. Esto, coincide con el hecho de haber recogido estados larvarios de Coenagrion cae- 
rulescens recien eclosionados en el área de estudio, aunque en este caso, la disminución del número de sus depredadores también facilitaría un mayor desarrollo de su población.

En el otro extremo dę la clasificación las especies esporádicas no se encontraron en la comunidad de otoño de 1982, al igual que ocurre con la mayoría de las clasificadas como accesorias.

Finalmente, los resultados de la aplicación del índice de diversidad $(\mathrm{H})$ y el cálculo de la diversidad máxima (Hmax) para conocer el grado de estabilidad de la comunidad, se muestran en la figura 6. Los valores más bajos, coinciden con los días posteriores a los picos de caudal y en el resto las mayores o menores oscilaciones dependen de la intensidad del flujo de agua. El $11 .^{\circ}$ día de muestreo, se alcanza el máximo valor de diversidad específica con $\mathrm{H}=3,88$ bits, superior incluso al obtenido para la comunidad de otoño de 1982 (tabla 2).

\section{DISCUSIÓN}

Algunos autores (Hynes, 1970; SiEgFrIEd \& KNIGHT, 1977) consideran que los ríos sometidos a riadas muestran una fauna bentónica pobre en especies y escasa en individuos. Sin embargo, esto no parece ser generalizable a todos los casos (CusHING \& GAINES, 1989) a juzgar por la variedad y densidad de las poblaciones de organismos bentónicos registradas en la Rambla del Moro. Probablemente, las diferencias más importantes con respecto a otros ríos, radiquen en la composición cualitativa y en el grado de equilibrio de la comunidad. Así, mientras que los Efemerópteros, Tricópteros y especies reófilas de Dípteros, dominan ampliamente la comunidad de ríos, en el caso de la Rambla del Moro, son los Dípteros, Heterópteros, Coleópteros y Odonatos los más importantes en cuanto a su contribución a la riqueza específica y abundancia. Esto resulta, además, totalmente coincidente con los resultados obtenidos para el río Le Rdat (Marruecos) por B ADRI et al. (1987) o para el Sycamore Creek (Arizona) por GRAY (1981) y FISHER et al. (1982), ambos de características semiáridas y áridas y que sufren riadas con cierta periodicidad.

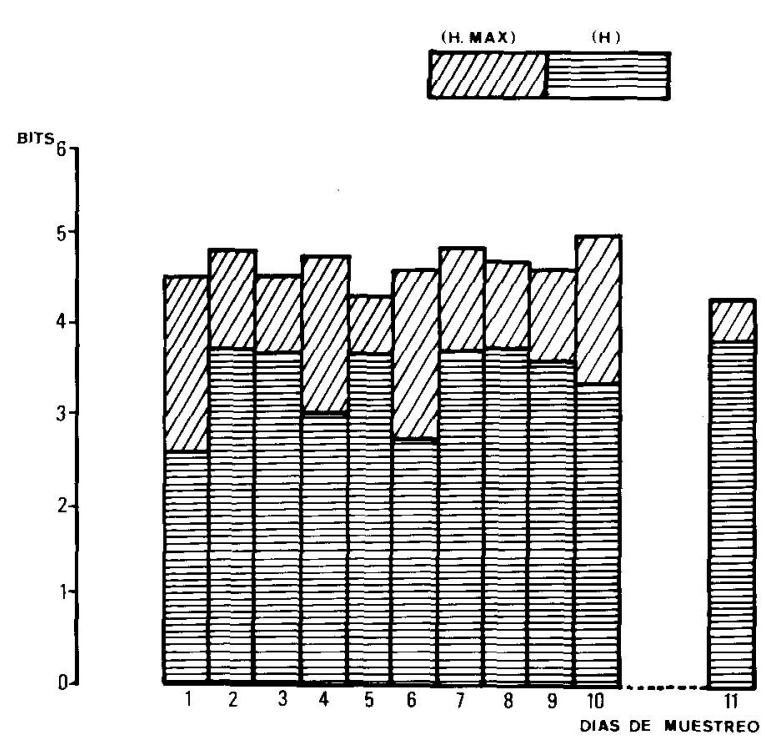

Figura 6. - Histogramas de diversidad (H) y diversidad máxima (Hmax), para cada muestra después de la riada de 1986. Diversity and maximum diversity values of the 11 day samples.

Los índices empleados en este estudio para describir la estructura de la comunidad de invertebrados acuáticos de la Rambla del Moro, antes de producirse la riada, muestran ciertas contradicciones, útiles de comentar, en relación con la tipología de las especies presentes (OrTEgA, 1988) que, en general, parecen quedar clasificadas como eurióicas y oportunistas mientras que la comunidad se muestra bastante equilibrada en relación a las curvas de dominancia (fig. 3) o a los valores de diversidad (tabla 2). Quizás, el uso de todos estos adjetivos, referidos a las especies o a las comunidades, debiera restringirse a los casos de organismos colonizadores procedentes de otros hábitats, pero no a las especies que, de alguna manera, parecen tolerar e incluso «interiorizar», los efectos de las perturbaciones. El resultado puede traducirse en un «equilibrio inestable» de la comunidad sometida a perturbaciones periódicas y, de hecho, Fisher \& GRIMm (1988) hablan de éstas como de elementos «organizadores» que limitan su grado de complejidad.

La riada que sufrió la Rambla del Moro en octube de 1986, eliminó el $50 \%$ de los taxones, en relación con la comunidad de otoño de 1982, to- 
Tabla 4. - Tabla comparativa de diferentes variables relativas a las avenidas de agua, composición de las comunidades de invertebrados acuáticos y los mecanismos adaptativos que utilizan, en tres ríos de características ambientales distintas que sufren riadas. Comparative table of different characteristics of floods, composition of invertebrate aquatic community and adaptative mechanisms. in three different strcams where floods occur.

\begin{tabular}{|c|c|c|c|}
\hline & $\begin{array}{l}\text { SYCAMORE CREEK } \\
\text { (Arizona) }\end{array}$ & $\begin{array}{l}\text { LE RDAT } \\
\text { (Marruecos) }\end{array}$ & $\begin{array}{l}\text { RAMBLA DEL MORO } \\
\text { (España) }\end{array}$ \\
\hline Tipologia del sistema & Río árido del desicrto & Río del Mediterráneo árido & Río del Mediterráneo semiárido \\
\hline Intensidad de las riadas & Media & Media - Alta & Variable \\
\hline Frecuenciu de las riadas & $\begin{array}{l}\text { Alta (invierno. primavera. } \\
\text { verano) }\end{array}$ & Media (primavera) & Media - Baja (otoño) \\
\hline $\begin{array}{l}\text { Grado de predecibilidud de las } \\
\text { riadas }\end{array}$ & Alta & Alta & Media \\
\hline $\begin{array}{l}\text { Ordenes de invertebrados mas } \\
\text { importantes }\end{array}$ & $\begin{array}{l}\text { Colcóptcros. Dípteros. } \\
\text { Tricóptcros, Hetcrópteros }\end{array}$ & $\begin{array}{l}\text { Dípteros, Coleópteros, } \\
\text { Tricópteros, Efcmerópteros }\end{array}$ & $\begin{array}{l}\text { Díptcros, Coleópteros, } \\
\text { Heterópteros, Odonatos }\end{array}$ \\
\hline Riqueza especifica & 48 taxones & 38 taxones & 61 taxones \\
\hline $\begin{array}{l}\text { Grado de resistencia de las } \\
\text { comunidades }\end{array}$ & Bajo & Bajo & Medio \\
\hline $\begin{array}{l}\text { Grado de resilencia de las } \\
\text { comunidades }\end{array}$ & Alto & Medio - Alto & Medio \\
\hline $\begin{array}{l}\text { Vías de recolonización mas } \\
\text { importantes }\end{array}$ & Ovoposición de adultos & $\begin{array}{l}\text { Deriva y movimientos desde la } \\
\text { llanura de inundación }\end{array}$ & $\begin{array}{l}\text { Deriva, movimientos desde la } \\
\text { llanura de inundación y } \\
\text { ovoposición de adultos }\end{array}$ \\
\hline $\begin{array}{l}\text { Estrategias adaptativas de las } \\
\text { especies }\end{array}$ & $\begin{array}{l}\text { Ciclos de vida cortos } \\
\text { Reproducción estacional } \\
\text { Sustitución temporal de especies }\end{array}$ & & $\begin{array}{l}\text { Ciclos de vida de duración } \\
\text { variable. Individuos de pequeño } \\
\text { tamaño (?). No sustitución de } \\
\text { especies }\end{array}$ \\
\hline
\end{tabular}

mada como referencia. Una riada de similares características ocurrida en el río Le Rdat eliminó el $76,32 \%$ de los taxones (BADRI et al., 1987) y Gray (1981) estima una pérdida media del $86 \%$ de los taxones para nueve riadas acaecidas, desde agosto de 1977 a octubre de 1979 en el Sycamore Creek.

Si el grado de resistencia a una perturbación (entendida como el cambio que ésta induce sobre el sistema) se puede medir como el porcentaje de cambio en, por ejemplo, la riqueza específica o la densidad de invertebrados (Fisher \& Grimm, 1988), entonces, los organismos de la Rambla del Moro, muestran una resistencia «media», dado que menos de la mitad de los taxones existentes, se ven afectados por la riada.

Transcurridos 27 días de la onda de crecida, la curva de frecuencias secuenciales de los taxones presentes en la Rambla del Moro (fig. 4), y el valor de diversidad (fig. 6), parecen indicar un alto grado de equilibrio y estabilidad en la comunidad. Esto no debe ser probable ya que sólo persisten 19 taxones y la abundancia total es de 87 individuos, la menor del estudio. Sin embargo, estos taxones son precisamente, los clasificados como fundamentales y constantes. El proceso de restablecimiento de la comunidad parece producirse de forma rápida por cuanto que, a los 27 días de la onda de crecida, se hallaban presentes, alrededor de un $45 \%$ de las especies de la comunidad de referencia. Fisher et al. (1982) obtienen tiempos de recuperación de la comunidad comprendidos entre 30 y 90 días, dependiendo de la estación del ano, sin embargo, Molles (1985), para un río de Nuevo Méjico, cuyas riadas se producen de forma no periódica, obtiene períodos de tiempo de hasta un ano para reconocer el restablecimiento de la comunidad. Así pues, en este sentido, y entendiendo la resilencia como una medida de la velocidad de recuperación de la comunidad tras producirse una perturbación, en el caso de la Rambla del Moro puede ser considerada como de grado «medio», más aún cuando el proceso de restablecimiento estuvo interrumpido por importantes variaciones en el caudal (fig. 2).

En relación con las estrategias que presentan las especies sometidas a este tipo de perturbaciones, son escasos los estudios que aportan datos sobre 
su número y tipología. Gray (1981), tras analizar aspectos sobre la biología de las especies más habituales en el Sycamore Creek, enumera distintos comportamientos adaptativos como estrategias seguidas por los insectos que habitan los ríos que sufren avenidas de agua. Ciclos de vida cortos, reproducción estacional, sustituciones temporales de unas especies por otras y, sobre todo, la interpretación de que los adultos voladores son los únicos estados de resistencia a las riadas, son algunas de las estrategias mencionadas por este autor. Recientemente, diferentes autores han mostrado la importancia del hiporreos como vía de recolonización y como estrategia adaptativa de muchos invertebrados de ríos que sufren riadas (ver revisión de Cushing \& Gaines, 1989).

Aunque no se conocen con precisión los ciclos de vida y las estrategias adaptativas de los invertebrados acuáticos de la Rambla del Moro, éstos parecen presentar una gran variedad de tipos de desarrollo. Por ejemplo, en el caso de los Heterópteros, Cymatia rogenhoferi presenta una sola generación al año y la forma adulta pasa por un período de latencia o hivernación (TAMANinI, 1979). En contraste, Heliocorisa vermiculata presenta, al menos, cuatro generaciones anuales y los adultos de otoño son típicamente voladores (VELASCO et al., 1990). Otras especies, como Cloeon dipterum o Anax imperator, manifiestan una cierta capacidad para acelerar sus tasas de desarrollo larvario. En general, no se ha observado una tendencia generalizada a acortar los ciclos de vida ni a que aparezcan sustituciones de las especies, afectadas por la riada, por otras más resistentes u oportunistas (tabla 1). No obstante, la mayoría de los insectos recolectados en la rambla presenta un tamaño más pequeño de lo que, habitualmente, se describe en la literatura, lo que podría interpretarse como una adaptación a estos tipos de perturbaciones. Además, ha quedado demostrada la importancia de los adultos voladores en el proceso de restablecimiento de la comunidad, después de producirse la riada (ORTEGa et al., 1991).

Como indican algunos autores (Power et al., 1988; Resh et al., 1988), los efectos de las riadas sobre los ecosistemas acuáticos, dependen tanto de su magnitud como de su frecuencia, en el sentido de que la primera, probablemente, está más relacionada con la capacidad o grado de resistencia de la comunidad, mientras que la segunda lo está con el grado de resilencia. En este sentido, cabe pensar que no todos los ríos que sufren riadas presentan mecanismos compensatorios similares. En un intento por sintetizar las diferencias en algunos de los aspectos más importantes que caracterizan a distintos ríos que sufren riadas, se ha elaborado la Tabla 4 en la que se comparan tres ríos de distintas características ambientales, expresando de forma cualitativa, algunas variables relativas al fenómeno de las riadas, a los principales efectos que provocan en las comunidades acuáticas y algunos de los mecanismos compensatorios detectados. En general, se observa cómo existe una tendencia, por parte de las especies, a diversificar sus mecanismos adaptativos y estrategias recolonizadoras, para amortiguar los efectos de estas perturbaciones, en relación con el grado de predecibilidad de las mismas. En cualquier caso, el desarrollo de futuros trabajos que pongan en práctica una metodología para cuantificar estos aspectos, permitirá conocer, de forma más precisa, las verdaderas relaciones existentes entre el carácter predecible de una perturbación, como las riadas, y el grado de «interiorización» de sus efectos por los ecosistemas a nivel de su composición y estructura bióticas.

\section{Bibliografía}

Badri, A.. J. Giudicelli \& G. Prevot, 1987. Effects d'une crue sur la communaute d'invertébrés benthiques d'une riviere méditerranéenne, Le Rdat (Maroc). Acta oecologica oecol. Gener., Vol. 8, n." 4: 481-500.

Bishop, J.E., 1973. Limnology of a small Malayan river Sungai Gombak. W. Junk, the Hague.

Cushing, C.E. \& W.L. GaInEs, 1989. Thoughts on recolonization of endorheic cold desert spring streams. J. N. Am. Benthol. Soc., 8 (3): 277-287.

Fisher, S.G., L.J. Gray, N.B. Grimm \& D.E. BusCh, 1982. Temporal succession in a desert stream ecosystem following flash flooding. Ecol. Monog. 52 (1): 93-100.

FISHER, S.G. \& N.B. GRIMM. 1988. Disturbance as a determinant of structure in a Sonoran Desert stream ecosystem. Verh. Internat. Verein. Limnol., 23: 1183-1189.

GASCHIGNARD, O.. 1984. Irnpact d'une crue sur les macroinvertebres benthiques d'un brass du Rhone. Verh. Internat. Verein. Limnol. 22: 1997-2001.

Giberson, D.J. \& R.J. HALl, 1988. Seasonal variation in faunal distribution within the sediments of a Canadian Shield stream, with emphasis on responses to spring floods. Can. J. Fish. Aquat. Sc., 45 (11): 1994-2002. 
GraY, L.J., 1980. Recolonization pathways and community development of desert stream macroinvertebrate. Disertation: Arizona State University.

GraY, L.J., 1981. Species composition and life histories of aquatic insects in a Lowland Sonoran Desert stream. Amer. Midland Natur. 106: 229-242.

Gray, L.J. \& S.G. Fisher. 1981. Posflood recolonization pathways of macroinvertebrates in a Lowland Sonoran Desert Stream. Amer. Midland Natur. 106 (2): 249-257.

Grimm, N.B. \& S.G. FISHER, 1989. Stability of periphyton and macroinvertebrates to disturbance by flash floods in a desert stream. J.N. Am. Benthol. Soc. 8 (4): 293-307.

HOOPER, F.F. \& D.R. OTTEY, 1988. Responses of macroinvertebrates of two headwater strearns to discharge fluctuations. Verh. Internar. Verein. Limnol. 23, 1159-116.

Hynes, H.B.N., 1970. The Ecology of running waters. Univer sity of Toronto Press, Toronto.

Jones, J.R.E., 1951. An ecological study of the River Towy. Anim. Ecol. 20: 68-86.

KRAFT, K.J. \& N.D. MUNDAHL, 1984. Effects of intermittent flow regulation on temperature and macroinvertebrate distribution and abundance in a Michigan River. Freshwat. In vertebr. Biol. 3 (1): 21-35.

Margalef, R., 1977. Ecología. Omega. Barcelona.

Margalef, R., 1983. Limnología. Omega. Barcelona.

MAY, R.M., 1975. Patterns of species abundance and diversity. In: CoDY \& DiAmond (Eds.) Ecology and evolution of communities: 81-120. Harvard Univ. Press. Cambridge, Mass.

McLAy, C.L., 1968. A study of drift in the Kakanui river, New Zealand. Austr. J. Mar. Freshwat. Res., 19: 139-149.

Millan, A.. 1985. Los Heterópteros acuáticos (Gerromorpha y Nepomorpha) de la Cuenca del Río Segura. S.E. de Espa$\tilde{n} a$. Tesis de Licenciatura. Universidad de Murcia.

Minshall, G.W. \& R.C. Petersen, 1985. Towards a theory of macroinvertebrate community structure in a stream ecosystems. Arch. Hydrobiol. 104 (1): 49-76.

Molles, M.D., 1985. Recovery of a stream invertebrate community from a flash flood in Tesuque Creek, New Mexico. Sothwestern Naturalist, 30: 279-287.

Montes, C., 1980. Las taxocenosis de Odonatos, Heterópteros y Coleópteros acuáticos en el área del Bajo Guadalquivir: Estructura y variación estacional de sus poblaciones y ambiente físico. Tesis doctoral. Universidad de Sevilla.

Neckles, H.A., H.R. Murkin \& J.A. Cooper; 1990. Influences of seasonal flooding on macroinvertebrate abundance in wetland habitats. Freshwater Biology 23: 311-322.

Ortega, M., 1988. La Rambla del Moro (Cuenca del Río Segura). Ambiente físico, biológico y alteraciones producidas por una riada. Tesis de Licenciatura. Universidad de Murcia.

Ortega, M., M.R. Vidal-Abarca, M. Suárez, J.L. GonZÁLEZ-BESERAN \& L. RAMíreZ-DIAZ, 1988. Características físico-químicas de las aguas superficiales de la Rambla del Moro después de una riada (Cuenca del Río Segura, S.E. de España). Limnética 4: 19-26.
Ortega, M., M.L. Suárez, M.R. Vidal-Abarca, R. GóMEZ \& L. RAMírEz-DíAz (1991). Aspects of postflood recolonization of macroinvertebrates in a «rambla» of southeast Spain («Rambla del Moro»: Segura River Basin). Verh. Internat. Verein. Limnol.

Power, M.E., R.J.Stout, C.F. Cushing, P.P. Haroer, F.R. Haver, W.J. Matthews, P.B. Moyle, B. Statzner \& 1.R. WAIS DE BADGEN, 1988. Biotic and abiotic controls in river and stream communities. J.N. Am. Benthol. Soc. 7 (4): 456-478.

Prevot, G. \& R. Prevot, 1986. lmpact d'une crue sur la communaute d'invertebres de la Moyenne Durance. Role dc la derive dans la reconstitution du peuplement du chenal principal. Annls. Limnol. 22 (1):89-98.

REICE, S.R., 1985. Experimental disturbance and the maintenancc of species diversity in a strcam cornmunity. Oecologia 67: 90-97.

Resh, V.H., A.V. Brown, A.P. Covich, M.E. Gurtz, H.W. Li, G.W. Minshall, S.R. Reice, A.L. Sheldon, J.B. WALLACE \& R. WISSMAR, 1988. The role of disturbance in stream ecology. J.N. Am. Benthol. Soc. 7 (4): 433-455.

Ricklefs, L., 1979. Ecology. Chiron, New York.

Routledge, R.D., 1980. The form of species-abundance distributions. J. Theor. Biol. 82: 547-558.

Santos, A., 1983. Biología y ecología de dos comunidades mediterráneas de coloeópteros terrestres de superficie. Tesis doctoral. Universidad de Murcia.

Shannon, C.E.\& W. WEAVER, 1949. The mathematicaltheory of communication. Univ. Illinois Press.

Scullion, J. \& A. SinTon, 1983. Effects of artificial freshets on substratum composition, benthic invertebrate fauna and invertebrate drift in two impounded rivers in mid-Wales. Hydrobiologia, 107: 261-269

Siegfried, C.A. \& A.W. KNight, 1977. The effect of washout in a Sierra foothill stream. Amer. Midland Natur., 98: 200-207.

SOLER, A. (coordinador), 1984. Estudio y directrices para el saneamiento del Río Segura (Prospección, estado actual previsiones y bases). 6 Volúmenes. Comunidad Autónoma de la Región de Murcia. Universidad de Murcia.

Tamanini, L., 1979. Guide per il riconoscimento delle specie animali delle acque interne italiane, 6. Eterotteri aquatici (Heteroptera: Gerromorpha \& Nepomorpha). Consiglio Nazionale delle Richerche.

THIERY, A., 1978. Etude des communates des invertebres aquatiques dans differents biotopes des marais du Plan du Bourg. These de specalite. Ecologie. Univ. de Droit d'economie et des Sciences Marseille.

Turcotte, P. \& P.P. HARPer, 1982. Drift patterns in a high Andean stream. Hydrobiologia, 89: 141-151.

Velasco, J., A. Millan \& N. Nieser, 1990. Observaciones sobre la colonización y el ciclo de vida de Heliocorisa ver miculata (PuTON, 1874) (Heteróptera, Corixidae) en pequeños estanques del SE español. Limnética, 6: 101-108.

WhitTaker, R., 1975. Communities and Ecosystems. NewYork: McMillan. 